\title{
EVALUATION OF OBSTETRIC NEAR MISS \& MATERNAL DEATHS IN A TERTIARY CARE TEACHING HOSPITAL
}

KEY WORDS: Maternal Near Miss , Maternal Deaths, Maternal Near Miss Incidence Ratio.

\section{Dr. B. Harika}

\section{Dr. Bhavishya Gollapalli*}

M.S, OBGY, Associate Professor, Dept Of Obstetrics And Gynaecology, Narayana Medical College And Hospital, Nellore.

Post Graduate, Dept of OBGY,Narayana Medical College and Hospital, Nellore. *Corresponding Author

Background: Maternal Mortality is a critical indicator to assess the quality of services provided by a health care system. The concept of severe acute maternal morbidity (SAMM) has been found to complement the assessment of maternal health services, since pregnant women's health status is not reflected by mortality indicators alone. Maternal near miss cases are more common than maternal deaths. Methods: A Retrospective analysis of maternal near miss cases and maternal deaths was undertaken in the Department of Obstetrics and Gynaecology, Narayana Medical College and Hospital, Nellore, Andhra Pradesh over a period of two years. This study was conducted to find out maternal near miss and maternal deaths. Results: There were 2000 deliveries, 66 MNM cases and 2 maternal deaths during the study period. The Maternal Near Miss Incidence Ratio (MNMIR) ratio was 33\%.The maternal near miss to mortality ratio was 33\% for each maternal death. The Maternal Mortality Ratio (MMR) was 100 per 1 lakh live births in our study. Conclusion: We suggest near miss approach to evaluate and improve the quality of care provided by the health care system. By reviewing the near miss cases we can learn about the processes and their deficiencies that are in place for the care of pregnant women.

\section{INTRODUCTION}

Maternal Mortality is a critical indicator to assess the quality of services provided by a health care system. The concept of severe acute maternal morbidity (SAMM) has been found to complement the assessment of maternal health services, since pregnant women's health status is not reflected by mortality indicators alone. Maternal near miss cases are more common than maternal deaths. The major reasons and causes are the same for MNM and maternal death rate (MDR). So review of MNM cases is likely to yield valuable information regarding severe morbidity which could lead to death of the mother, if not intervened properly and in time (WHO).

WHO in 2009 has come up with certain criteria for identification of these cases so as to address the need for a consensus which can be used worldwide. These markers include clinical signs, laboratory tests and management parameters for identifying MNM cases.

\section{MATERIAL AND METHODS :}

A Retrospective analysis of maternal near miss cases and maternal deaths was undertaken in the Department of Obstetrics and Gynaecology, Narayana Medical College and Hospital, Nellore, Andhra Pradesh over a period of two years. This is a tertiary care teaching institution which provides antenatal care and delivery services for low risk as well as high risk pregnant women. This study was conducted to find out maternal near miss and maternal deaths. For identification of maternal near miss cases, WHO 2009 criteria was taken into consideration.

Potential life threatening conditions were diagnosed and demographic characteristics, gestational age at the time of morbidity, mode of delivery, ICU admissions, duration of ICU stay, need for transfusion and surgical interventions to save the life of the mother was noted. Maternal mortality during the study period was also analysed.The following indices were calculated:

1. Maternal near miss incidence ratio (MNMIR).

2. Maternal near miss to mortality ratio.

3. Mortality index.

4. Maternal mortality ratio(MMR).

\section{RESULTS:}

There were 2000 deliveries, 66 MNM cases and 2 maternal deaths during the study period. The Maternal Near Miss
Incidence Ratio (MNMIR) ratio was 33\%.The maternal near miss to mortality ratio was $33 \%$ for each maternal death. The Maternal Mortality Ratio (MMR) was 100 per 1 lakh live births in our study.Majority of them were in the age group of 25 to 35 years $(74.2 \%)$. Two maternal deaths were among this age group. In maternal deaths, 1 was primipara and 1 was multipara. Among MNM cases, 26 (40\%) were multipara and $40(60 \%)$ cases were primipara. All 2 maternal deaths occurred postnatally. Among MNM cases, 8 (12\%) were less than 12 weeks, $3(5 \%)$ were between $13-28$ weeks, $8(12 \%)$ were above 28 weeks and remaining 47 (71\%) were postnatal cases. In MNM cases 11 were booked and 55 were unbooked, where as 38 cases were referred from outside hospitals.

The number of cases who required ICU care were 18. Among those admissions, the most common cause of admissions were due to Gestational HTN (5), haemorrhage (6). Around 14 cases were stayed in the ICU for period of $1-5$ days and 3 cases stayed for 6 - 10 days. 24 cases undergone blood transfusions. Around 40 underwent LSCS and 13 cases vaginal delivery. Thirty one cases had a history of organ failure most commonly haematological (22). Hypertension (27 cases) and haemorrhage due to ectopic, abortion, APH, PPH (21 cases) were most common cause of MNM.

On evaluating the primary obstetric complication leading to MNM, hypertensive disorders of pregnancy (51\%) were the commonest complications. Haemorrhage accounted for $39 \%$ cases of MNM which included ruptured ectopic pregnancy , incomplete abortions, rupture uterus, APH and PPH. Among the indirect causes cardiac diseases accounted for $7 \%$. In our study there were 2 maternal deaths and maternal mortality ratio was 100 per $1,00,000$ live births. There were one maternal death due to hypertensive disorder of pregnancy and one due to haemorrhagic disorder.

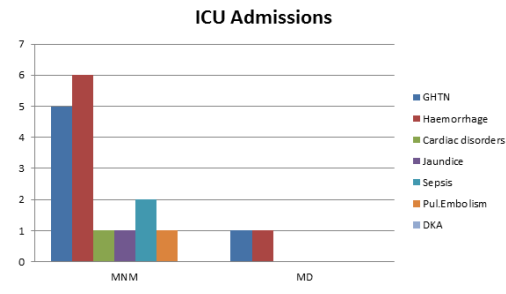

Chart No:1 Classification according to cause of ICU admissions: 


Table No: 1 Duration of ICU stay among MNM and MD
\begin{tabular}{|l|l|l|}
\hline Duration of ICU stay & MNM & MD \\
\hline $1-5$ days & 13 & 1 \\
\hline $6-10$ days & 2 & 1 \\
\hline$>10$ days & 1 & 0 \\
\hline
\end{tabular}

\section{MNM}

\section{- HTN Hemorrhage $\quad$ Sepsis Indirect causes}

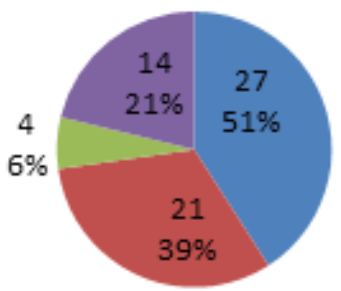

Chart No:2 Distribution of MNM events \& cause of MD: HTN n-27

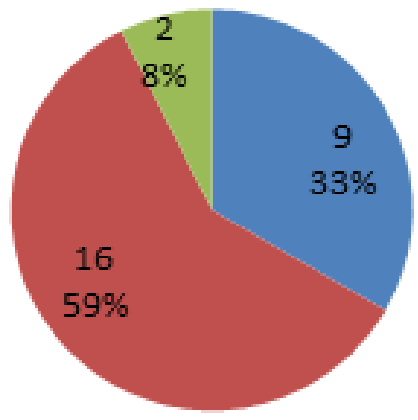

Pre eclampsia

Eclampsia

HELLP

\section{Haemorrhage $n=21$}

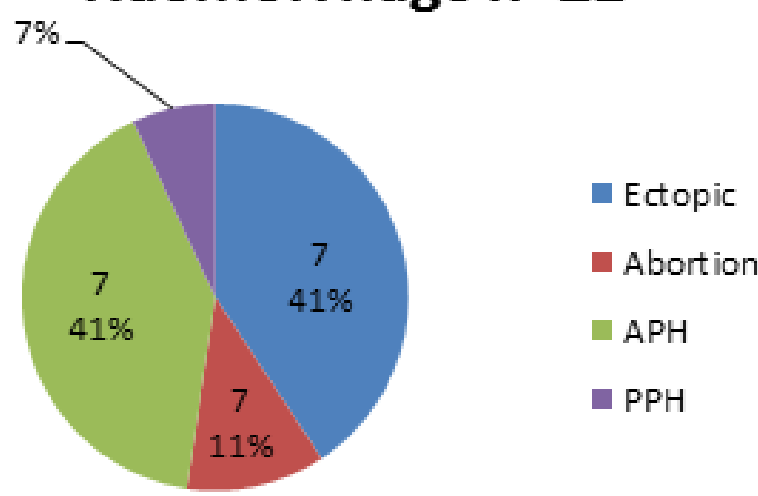

Chart No:3\& 4 Distribution of causes of HTN and Haemorrhage

\section{DISCUSSION}

A woman who survives life threatening conditions during pregnancy, abortion, and childbirth or within 42 days of pregnancy termination, irrespective of receiving emergency medical or surgical interventions, is called maternal near miss. The maternal near miss incidence ratio (MNMR) was $33 / 1000$ live births in our hospital. Several studies from the developing countries show the same trend and vary from anywhere between 15-40/1000 live births . Near miss ratios have been reported as 44.3 per 1000 in Brazil , 3.83 per 1000 in Scotland, and 34 per 1000 in aWHO survey .
The near miss to mortality ratio in our study was 33:1.This indicates better care of patients in our hospital. A Syrian study showed a near miss to mortality ratio of $60: 1$ and whereas some studies from Western Europe shows a ratio of 117-223:114. The causes of near miss vary in different geographical areas of the world and also there are variations within countries. Haemorrhage, hypertensive disorders, sepsis and obstructed labor are the most important causes in the developing countries. In our study, the main primary determinant of severe maternal morbidity was hypertensive disorders, eclampsia having a high prevalence of 16 cases.

But several studies have shown haemorrhagic disorders as the primary determinant of severe maternal morbidity. Haemorrhagic complications (mainly postpartum bleeding, rupture ectopic, and uterine rupture) were the second common cause for leading to near miss, accounting for $29.4 \%$ in our study. Maternal mortality is a critical indicator to assess the quality of services provided by a health care system. Every day, approximately nearly 830 women die from preventable causes related to pregnancy and childbirth mostly in low resource settings (WHO). The target is to reduce the global maternal mortality ratio to less than 70 per 100000 live births by 2030 , as part of the sustainable Development Agenda.

\section{CONCLUSION:}

- We suggest near miss approach to evaluate and improve the quality of care provided by the health care system.

- By reviewing the near miss cases we can learn about the processes and their deficiencies that are in place for the care of pregnant women.

\section{REFERENCES}

1. Evaluating the quality of care for severe pregnancy complications: the WHO near miss approach for maternal health. Geneva:World Health Organization; 2011.

2. L. Say, J. P. Souza, and R. C. Pattinson, "Maternal near miss-towards a standard tool for monitoring quality of maternal health care," Best Practice and Research, vol.23, no.3,pp. 287-296,2009.

3. Global Strategy for Women's, Children's and Adolescents' Health,2016 to 2030,New York United Nations 2015.

4. Galvao, Pereira, Mendonca et all, "The prevalence of severe maternal morbidity and near miss and associated factors in Sergipe, Northeast Brazil" BMC Pregnancy and Childbirth, vol 14, no 25,2014

5. V. Brace, G. Penney, and M. Hall, "Quantifying severe maternal morbidity: a Scottish population study,"BJOG, vol. 11 1, no.5, pp. 481-484,2004.

6. J. P. Souza, J. G. Cecatti, A. Faundes et al., "Maternal near miss and maternal death in the World Health Organization's 2005 global survey on maternal and perinatal health," Bulletin of the World Health Organization, vol. 88, no. 2, pp 113-119,2010.

7. J. van Roosmalen and J. Zwart, "Severe acute maternal morbidity in highincome countries," Best Practice and Research: Clinical Obstetrics and Gynaecology, vol.23,no.3,pp. 297-304,2009.

8. P. Souza, J. G. Cecatti, M. A. Parpinelli, S. J. Serruya, and E. Amaral, "Appropriate criteria for identification of near-miss maternal morbidity in tertiary care facilities: a cross sectional study," BMC Pregnancy and Childbirth, vol. 7,no.20,pp. 1-8,2007.

9. Roopa PS, Shailja Verma, Lavanya Rai, Pratap Kumar, Murlidhar V. Pai, and Jyothi Shetty, 'Near Miss Obstetric Events and Maternal Deaths in a Tertiary Care Hospital: An Audit,'Journal of Pregnancy. 